\title{
Spatial Attention Interacts With Serial-Order Retrieval From Verbal Working Memory
}

Psychological Science 24(9) 1854-1859

() The Author(s) 2013

Reprints and permissions:

sagepub.com/journalsPermissions.nav DOI: $10.1177 / 0956797613479610$ pss.sagepub.com

\author{
Jean-Philippe van Dijck ${ }^{1}$, Elger L. Abrahamse ${ }^{1}$, \\ Steve Majerus ${ }^{2}$, and Wim Fias ${ }^{1}$ \\ ${ }^{1}$ Department of Experimental Psychology, Ghent University, and ${ }^{2}$ Department of \\ Psychology: Cognition and Behavior, University of Liège
}

\begin{abstract}
The ability to maintain the serial order of events is recognized as a major function of working memory. Although general models of working memory postulate a close link between working memory and attention, such a link has so far not been proposed specifically for serial-order working memory. The present study provided the first empirical demonstration of a direct link between serial order in verbal working memory and spatial selective attention. We show that the retrieval of later items of a sequence stored in working memory-compared with that of earlier itemsproduces covert attentional shifts toward the right. This observation suggests the conceptually surprising notion that serial-order working memory, even for nonspatially defined verbal items, draws on spatial attention.
\end{abstract}

\section{Keywords}

attention, short-term memory

Received 8/3/12; Revision accepted 1/30/13

Working memory (WM) is a cognitive system that allows the brief maintenance of information in an active and accessible state in the mind, and it is thought to be crucial within such major domains as language, reasoning, and learning (e.g., Baddeley, 2012). Typically, across these domains, the relevant information is serially organized, and the maintenance of serial order has been recognized as a critical feature of WM (Marshuetz, 2005).

An increasing number of researchers have claimed that WM is strongly attention based, defending the view that WM emerges from attentional processes operating on long-term memory representations (e.g., Cowan, 1995; Postle, 2006). However, so far, the various models of WM for serial order (e.g., Burgess \& Hitch, 1999; Henson, 1998) have not followed up on this development and do not specifically emphasize attentional processes. Conversely, more general models of WM (e.g., Oberauer \& Hein, 2012) acknowledge an important role for attentional factors but do not explicitly account for serial order. In the current study, we aimed to bridge these literatures.

Spatial selective attention improves visual processing at attended versus unattended locations (e.g., Johnston,
McCann, \& Remington, 1995). Earlier studies have shown a link between spatial selective attention and visuospatial WM as stimulus processing improves at memorized locations compared with nonmemorized locations (e.g., Awh \& Jonides, 2001; Theeuwes, Kramer, \& Irwin, 2011). Moreover, Nobre et al. (2004) demonstrated that such spatial selective attention has an internal equivalent, in the sense that people can perform an attentional search over internal spatial representations-the latter being grounded in largely the same neural substrate as external spatial attention.

We hypothesized that serial-order WM is intrinsically linked to spatial attention: The selection of relevant information from a serial-order representation in WM is driven by an attentional search across an internal space.

\footnotetext{
Corresponding Authors:

Jean-Philippe van Dijck, Department of Experimental Psychology,

Ghent University, Henri Dunantlaan 2, 9000 Ghent, Belgium

E-mail: jeanphilippe.vandijck@ugent.be

Elger L. Abrahamse, Department of Experimental Psychology, Ghent University, Henri Dunantlaan 2, 9000 Ghent, Belgium

E-mail: elger.abrahamse@ugent.be
} 
Although this hypothesis is conceptually surprising, some indications for it exist. First, several studies have indicated the involvement of selective attention-although not specifically spatially defined-in the efficient maintenance of serial order in WM (e.g., Majerus et al., 2012; Majerus, Heiligenstein, Gautherot, Poncelet, \& Van der Linden, 2009). Second, it was recently demonstrated that brief sequences of words serially stored in WM are linked to space: Items from the beginning of such a sequence facilitated left-hand responses during the retention interval, whereas items from the end of the sequence facilitated right-hand responses, even when serial position was not relevant during retention (van Dijck \& Fias, 2011). Although this study clearly demonstrated a link between serial information and spatial processing, it does not allow any conclusion about the nature of this link and about whether it involves spatial attention per se.

To test our hypothesis, we explored the effect of the retrieval of serially stored items from WM on performance in a typical spatial-attention-cuing task (Posner, 1980; see Fig. 1a). Although both the verbal and spatial domains can involve serial order, the most convincing demonstration of such an effect would be with centrally presented verbal items (given that visuo-spatial information necessarily implies spatial processing). Three experiments were conducted using digits, which are frequently used in investigations of verbal WM. In each experiment, participants memorized four successively and centrally presented digits in the order of presentation (Phase 1). During the retention interval, they performed a speeded dot-detection task in which dots appeared randomly at the left or right sides of the screen (Phase 2). A digit was presented before each dot as a go/no-go cue; digits from the WM sequence cued go trials, whereas other digits cued no-go trials. Finally, serial-order knowledge was tested to ensure that the sequence was accurately maintained over the retention interval (Phase 3).

Because spatial-attention processes underlying WM strongly interface with external spatial attention (e.g., Awh \& Jonides, 2001; Nobre et al., 2004), we hypothesized that the retrieval of these serially stored WM items would be paralleled by shifts in external attention-based on WM position-that would subsequently enhance the perceptual processing of the dots appearing on the attended location. Thus, we predicted an increasing advantage in detecting right-side dots with increasing WM position.

\section{Experiment 1}

\section{Participants}

Twenty-four students (19 female, 5 male; mean age $=23$ years, $S D=3.13$; all native Dutch speakers) from Ghent University participated. ${ }^{1}$

\section{Stimuli and metbod}

An experimental session contained 36 blocks of three phases each. Phase 1 involved the central, self-paced serial presentation of four digits $\left(0.45^{\circ}\right.$; digits, which were pseudorandomly sampled from 1 to 8 were balanced across WM positions for Blocks 1-32 and randomly determined for the remaining 4 blocks). After a rehearsal period $(2,500 \mathrm{~ms})$, the speeded dot-detection task (Phase 2) started with the presentation of a central fixation cross $\left(0.25^{\circ}\right)$ centered between two rectangles $\left(1^{\circ} \times 0.67^{\circ} ; 3.20^{\circ}\right.$ eccentricity). After $250 \mathrm{~ms}$, a digit (uninformative about dot location) replaced the fixation cross for $300 \mathrm{~ms}$. Participants were instructed to fixate this central location throughout Phase 2. To optimize the time window for observing attentional modulation, we mixed cue-target intervals (CTIs) of 100, 250, and $400 \mathrm{~ms}$.

Next, the target $\left(\mathrm{a}\right.$ white dot; $0.5^{\circ} \times 0.5^{\circ}$ ) appeared in one of the rectangles for $150 \mathrm{~ms}$. To ensure WM access, we had participants respond only to trials on which digits from the WM sequence appeared (go trials), with a righthand key press on a response device aligned to body midline. The response deadline and intertrial interval (ITI) were 700 and $250 \mathrm{~ms}$, respectively. To discourage anticipatory responding, we considered trials with a response during dot presentation $(<150 \mathrm{~ms})$ to be noresponse trials (no response was recorded and the ITI was initiated only after a 700-ms penalty), and we included catch trials in which digit presentation was not followed by a target. Each block contained 20 trials. Each digit was presented twice followed by a dot; the memorized digits were additionally presented once, followed by a catch trial.

Finally, in Phase 3, we verified sequence maintenance with participants' responses to three questions about serial order (e.g., "Kwam 1 voor 8?", Dutch for "Was 1 preceded by 8?"). These questions were on the three possible pairs of subsequent WM items, the order of which either did or did not correspond to the order of the WM sequence (items were vertically arranged to avoid any horizontal association). The factors CTI, WM position, and dot location were fully crossed (12 measurements per condition).

\section{Results and discussion}

Trials from WM sequences with accurate serial-order verification (on average, 33.22 of 36 sequences) and correct go trials (accuracy on the dot-detection task was 94\%, 99\%, and $98 \%$ for the go, no-go, and catch trials, respectively) were considered. The mean reaction time (RT) was $314 \mathrm{~ms}(S D=46)$.

Each participant's mean RTs were computed for each condition and subjected to a $3 \times 4 \times 2$ repeated measures 
a

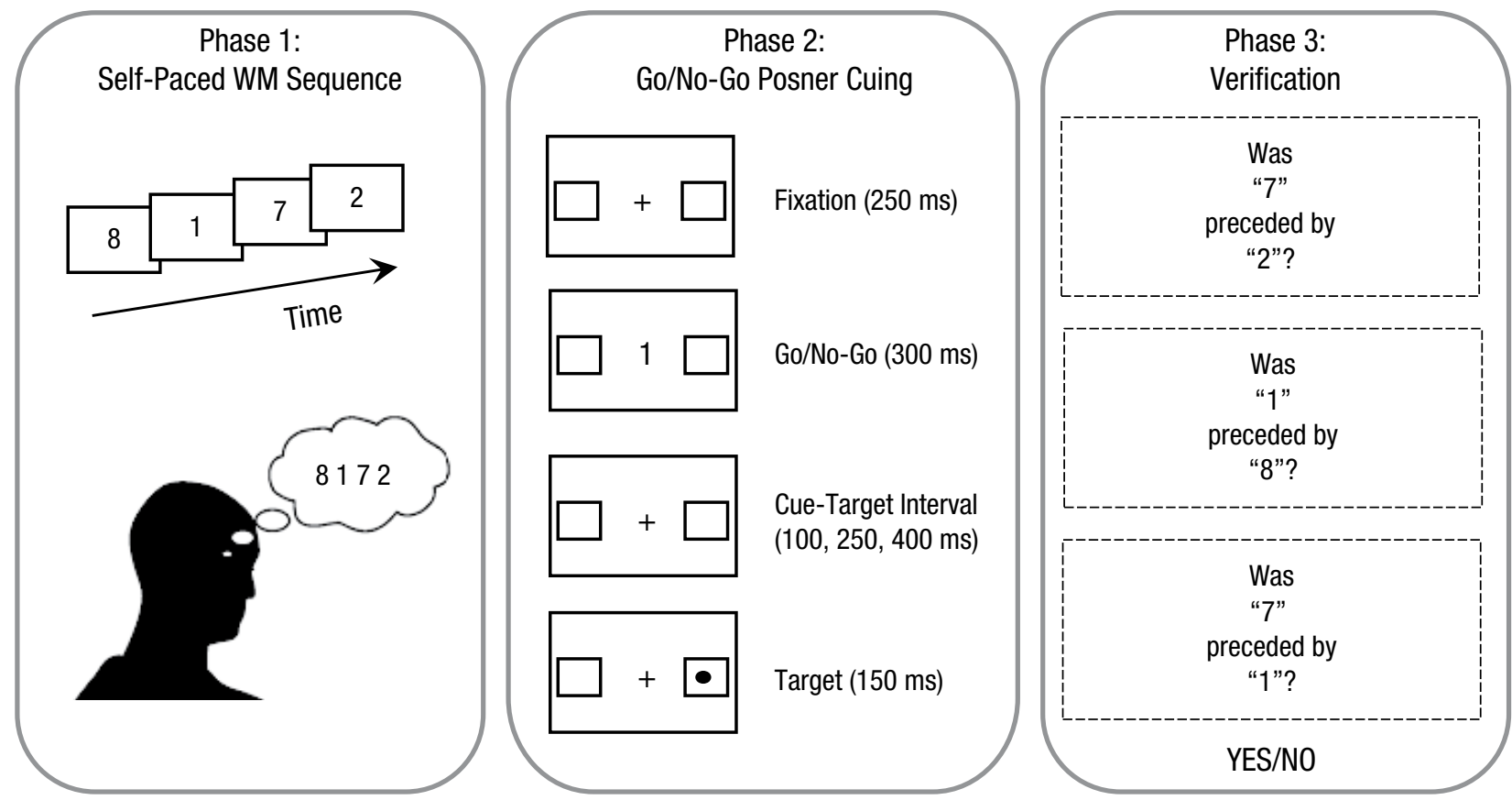

b

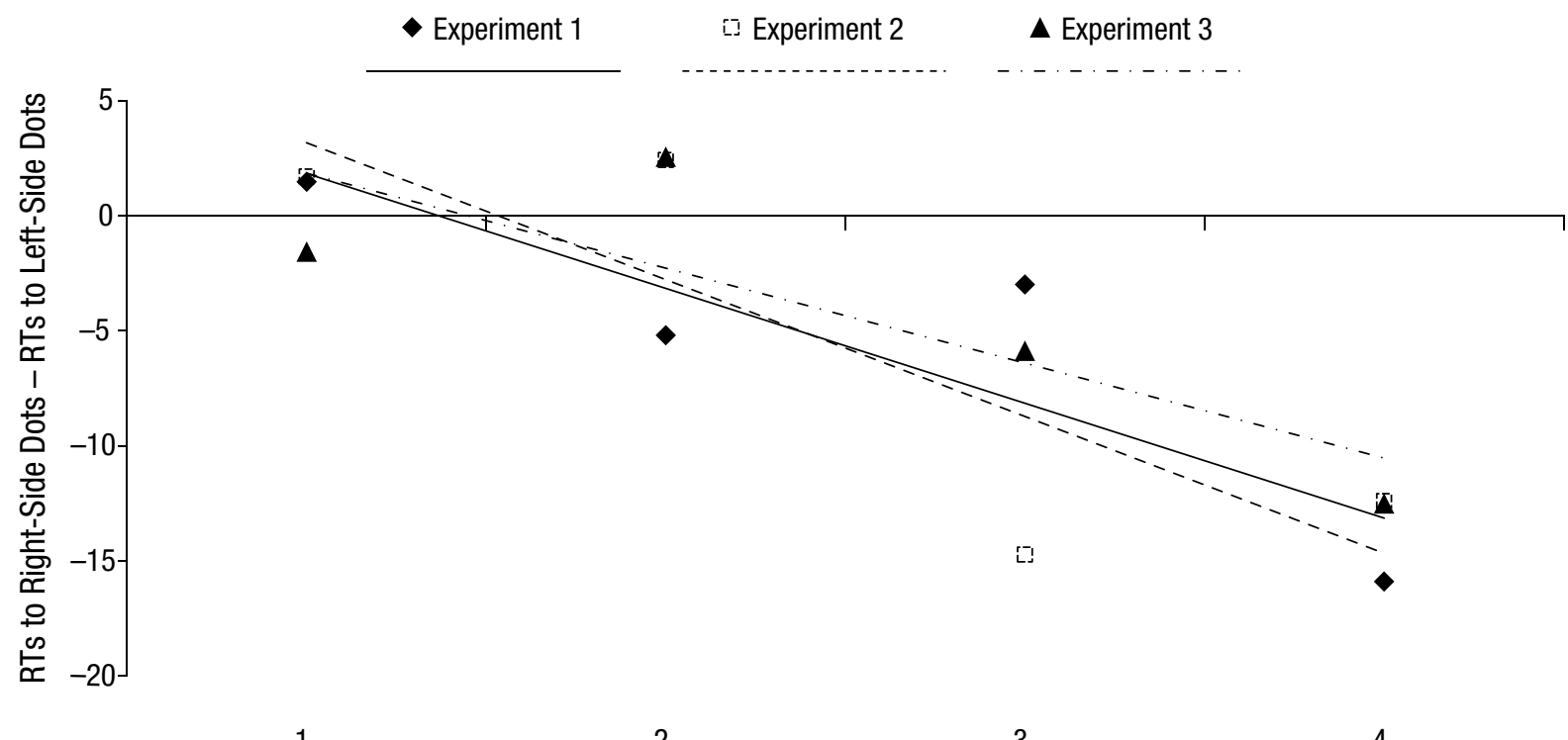

Position in WM

Fig. 1. Task design (a) and results (b) of Experiment 1. In each experimental session (a), Phase 1 involved the presentation of four digits from 1 to 8 . After a rehearsal period, Phase 2 began with the presentation of a central fixation cross centered between two rectangles. After $250 \mathrm{~ms}$, a digit replaced the fixation cross for $300 \mathrm{~ms}$; participants fixated this central location throughout Phase 2. Following a cue-target interval, the target appeared in one of the rectangles for $150 \mathrm{~ms}$. This series of events repeated for each trial in Phase 2. Finally, in Phase 3 , we verified sequence maintenance with participants' responses to three questions about the serial order of the presented numbers. The graph (b) shows differences between reaction times (RTs) to right- and left-side dots as a function of working memory (WM) sequence position and experiment.

analysis of variance (ANOVA) with CTI (100, 250, $400 \mathrm{~ms})$, WM position (1, 2, 3, 4), and dot location (left, right) as within-subjects variables (see Table 1). GreenhouseGeisser correction was applied when appropriate. This analysis revealed main effects of CTI, $F(1.44,33.14)=$ $15.55, p<.001, \eta_{p}^{2}=.403$, and WM position, $F(2.75$, 63.23) $=13.55, p<.001, \eta_{p}^{2}=.371$. A polynomial contrast indicated a linear increase, $F(1,23)=37.77, p<.001$, 
Table 1. Results from Experiment 1: Weighted Average Reaction Times for All Cue-Target Intervals and Conditions

\begin{tabular}{lcccc}
\hline $\begin{array}{l}\text { Cue-target } \\
\text { interval and } \\
\text { dot location }\end{array}$ & \multicolumn{4}{c}{ Working memory position } \\
\cline { 2 - 5 } & & 2 & 3 & 4 \\
\hline $100 \mathrm{~ms}$ & & & \\
$\quad$ Left side & $318 \mathrm{~ms}$ & $319 \mathrm{~ms}$ & $325 \mathrm{~ms}$ & $355 \mathrm{~ms}$ \\
Right side & $316 \mathrm{~ms}$ & $315 \mathrm{~ms}$ & $335 \mathrm{~ms}$ & $341 \mathrm{~ms}$ \\
$250 \mathrm{~ms}$ & & & & \\
Left side & $293 \mathrm{~ms}$ & $296 \mathrm{~ms}$ & $311 \mathrm{~ms}$ & $325 \mathrm{~ms}$ \\
Right side & $296 \mathrm{~ms}$ & $295 \mathrm{~ms}$ & $300 \mathrm{~ms}$ & $298 \mathrm{~ms}$ \\
$400 \mathrm{~ms}$ & & & & \\
Left side & $315 \mathrm{~ms}$ & $316 \mathrm{~ms}$ & $321 \mathrm{~ms}$ & $316 \mathrm{~ms}$ \\
Right side & $319 \mathrm{~ms}$ & $306 \mathrm{~ms}$ & $314 \mathrm{~ms}$ & $309 \mathrm{~ms}$ \\
\hline
\end{tabular}

$\eta_{p}^{2}=.622$, in the main effect of WM position, suggesting serial scanning. The CTI $\times$ WM Position interaction, $F(4.01,92.27)=4.12, p=.004, \eta_{p}^{2}=.152$, indicated that the linear increase was present only with CTIs of 100 $\mathrm{ms}, F(1,23)=35.74, p<.001, \eta_{p}{ }^{2}=.608$, and $250 \mathrm{~ms}$, $F(1,23)=14.18, p=.001, \eta_{p}{ }^{2}=.381$. In addition, the WM Position $\times$ Dot Location interaction, $F(2.39,54.95)=3.01$, $p=.049, \eta_{p}^{2}=.116$, indicated the crucial association between serial order in WM and spatial attention. A polynomial contrast of WM position in its interaction with dot location revealed a linear relationship, $F(1,23)=6.20$, $p=.020, \eta_{p}^{2}=.212$. The RT advantage in detecting rightside over left-side dots increased by an average of $4.99 \mathrm{~ms}$ per WM position (see Fig. 1b for RT differences per WM position), which confirmed our prediction that a digit's WM position modulates dot-detection performance.

\section{Experiment 2}

Although dot-detection performance is typically not driven by saccadic eye movements (Fischer, Castel, Dodd, \& Pratt, 2003; Pratt, Spalek, \& Bradshaw, 1999), Experiment 2 was conducted to ensure that the results obtained in Experiment 1 could be attributed to covert shifts of attention. We recorded the electrooculogram (EOG) to detect and exclude trials with horizontal saccades. Furthermore, it has previously been shown that numbers (when not explicitly memorized) can shift spatial attention (Fischer et al., 2003). To exclude the possibility that the interaction between WM position and dot location was driven by digit magnitude, we fully balanced digit magnitude across WM positions in Experiment 2.

\section{Participants}

Nineteen students (17 female, 2 male; mean age $=22$ years, $S D=2.60$; all native Dutch speakers) from Ghent University participated.

\section{Stimuli and metbod}

This experiment's stimuli, setup, and design were similar to those of Experiment 1, with the following exceptions: (a) Horizontal EOG was recorded using the BioSemi ActiveTwo system (BioSemi, Amsterdam, The Netherlands; electrodes placed on the left and right canthus); (b) 40 sequences were presented, allowing for numerical magnitude to be manipulated orthogonally to WM position; (c) CTIs were normally distributed around an average of $250 \mathrm{~ms}$ ( $S D=58$; visual inspection of the data from Experiment 1 indicated that the effect was most consistent at the 250-ms CTI, so in Experiment 2, we aimed to increase the number of observations without losing the temporal unpredictability of target onset); (d) because virtually no anticipatory responses were made in Experiment 1 (3 out of 6,337 trials), responses were allowed during dot presentation, followed by a regular ITI; and (e) the response deadline was set to $1,000 \mathrm{~ms}$, and the ITI was set to $50,150,250,350$, or $450 \mathrm{~ms}$ (ITIs were unpredictable on each trial and were presented an equal amount of times across the experiment) in order to optimize the design for continuous electroencephalogram recordings (not reported here). In total, 800 trials were performed, equally balanced across conditions.

\section{Results}

Correct go trials were considered (accuracy on the dotdetection task was 97\%, 99\%, and 98\% for the go, no-go, and catch trials, respectively) from correctly verified WM sequences (on average, 38.26 of 40 sequences) but only when no horizontal saccades (abrupt level deviations from baseline in the horizontal EOG, derived from visual inspection; occurred on 3\% of the correct go trials) occurred between the onset of the cue and the registration of the response. The mean RT was $357 \mathrm{~ms}$ $(S D=42)$.

Each participant's mean RTs were computed for each condition and subjected to a $2 \times 4 \times 2$ repeated measures ANOVA with digit magnitude $(\leq 4, \geq 5)$, WM position ( 1 , $2,3,4)$, and dot location (left, right) as within-subjects variables (see Table 2). This analysis revealed main effects of WM position, $F(2.02,36.34)=18.81, p<.001, \eta_{p}{ }^{2}=$ .511 , and dot location, $F(1,18)=7.52, p=.013, \eta_{p}^{2}=.295$. A linear trend was observed in the effect of WM position, $F(1,18)=29.57, p<.001, \eta_{p}^{2}=.622$. We replicated the crucial association between serial order in WM and spatial attention, as WM position interacted with dot location, $F(3,54)=4.03, p=.012, \eta_{p}^{2}=.183$. Again, a linear relationship was observed, $F(1,18)=5.02, p=.038, \eta_{p}^{2}=$ .218. The RT advantage in detecting right-side over leftside dots increased by an average of $5.94 \mathrm{~ms}$ per WM position (see Fig. 1b). No effects of digit magnitude were found, $p s>.170$, which indicated that the interaction 
Table 2. Results from Experiments 2 and 3: Weighted Average Reaction Times for All Dot-Magnitude and Dot-Location Conditions

\begin{tabular}{|c|c|c|c|c|}
\hline \multirow{2}{*}{$\begin{array}{l}\text { Digit magnitude } \\
\text { and dot location }\end{array}$} & \multicolumn{4}{|c|}{ Working memory position } \\
\hline & 1 & 2 & 3 & 4 \\
\hline \multicolumn{5}{|c|}{ Experiment 2} \\
\hline \multicolumn{5}{|l|}{ Small $(\leq 4)$} \\
\hline Left side & $348 \mathrm{~ms}$ & $345 \mathrm{~ms}$ & $361 \mathrm{~ms}$ & $386 \mathrm{~ms}$ \\
\hline Right side & $353 \mathrm{~ms}$ & $348 \mathrm{~ms}$ & $349 \mathrm{~ms}$ & $370 \mathrm{~ms}$ \\
\hline \multicolumn{5}{|l|}{ Large $(\geq 5)$} \\
\hline Left side & $350 \mathrm{~ms}$ & $346 \mathrm{~ms}$ & $369 \mathrm{~ms}$ & $372 \mathrm{~ms}$ \\
\hline Right side & $349 \mathrm{~ms}$ & $347 \mathrm{~ms}$ & $352 \mathrm{~ms}$ & $364 \mathrm{~ms}$ \\
\hline \multicolumn{5}{|c|}{ Experiment 3} \\
\hline \multicolumn{5}{|l|}{ Small $(\leq 4)$} \\
\hline Left side & $368 \mathrm{~ms}$ & $372 \mathrm{~ms}$ & $379 \mathrm{~ms}$ & $380 \mathrm{~ms}$ \\
\hline Right side & $368 \mathrm{~ms}$ & $373 \mathrm{~ms}$ & $372 \mathrm{~ms}$ & $373 \mathrm{~ms}$ \\
\hline \multicolumn{5}{|l|}{ Large $(\geq 5)$} \\
\hline Left side & $363 \mathrm{~ms}$ & $358 \mathrm{~ms}$ & $376 \mathrm{~ms}$ & $380 \mathrm{~ms}$ \\
\hline Right side & $361 \mathrm{~ms}$ & $362 \mathrm{~ms}$ & $371 \mathrm{~ms}$ & $363 \mathrm{~ms}$ \\
\hline
\end{tabular}

between WM position and dot location was independent from the numerical magnitude of the memorized numbers.

\section{Experiment 3}

In Experiments 1 and 2, only right-handed responses were used during dot detection. To exclude the possibility that the effects from these experiments were mere accidental side effects of right-hand responding, we conducted a final experiment with vocal responses. This response modality has the additional advantage that it is not spatially defined (as is the case with manual responses) and, thus, does not provide any hint for spatial coding by itself.

\section{Participants}

Twenty-one students (12 female, 9 male; mean age $=23$ years, $S D=4.51$; all native Dutch speakers) from Ghent University participated.

\section{Stimuli and method}

This experiment's stimuli, setup, and design were similar to those of Experiment 2 except that during dot detection, vocal responses were used ("ja," Dutch for "yes"). Because of technical limitations of the voice-key device, only RTs of less than $801 \mathrm{~ms}$ were recorded.

\section{Results}

Trials from WM sequences with accurate serial-order verification (on average, 37.19 of 40 sequences) and correct go trials (accuracy on the dot-detection task was 94\%, $99 \%$, and $99 \%$ for the go, no-go, and catch trials, respectively) were considered. The mean RT was $370 \mathrm{~ms}$ $(S D=59)$.

A $2 \times 4 \times 2$ repeated measures ANOVA with digit magnitude $(\leq 4, \geq 5)$, WM position $(1,2,3,4)$, and dot location (left, right) as within-subjects variables (see Table 2 ) revealed main effects of digit magnitude, $F(1,20)=12.29$, $p=.002, \eta_{p}{ }^{2}=.381$, and WM position, $F(2.14,42.77)=$ $3.91, p=.025, \eta_{p}^{2}=.163$. A linear trend was observed in the effect of WM position, $F(1,20)=7.17, p=.014, \eta_{p}^{2}=$ .264. The interaction between WM position and dot location was significant, $F(3,60)=3.11, p=.033, \eta_{p}^{2}=.134$. Again, a linear relationship was observed, $F(1,20)=9.63$, $p=.006, \eta_{p}{ }^{2}=.325$. The RT advantage in detecting rightside over left-side dots increased by an average of 4.12 ms per WM position (see Fig. 1b), replicating the observation that a digit's WM position modulates the speed of dot detection in a context in which no manual responses were required. Interactions with digit magnitude were not significant, $p s>.40$.

\section{General Discussion}

The relative speed of detecting left- and right-side dots in a selective-attention task was modulated by the retrieval of serially organized verbal items in WM. Specifically, the later the position being retrieved from WM during the dot-detection task, the faster the dot-detection response for right-side relative to left-side dots. This is the first empirical demonstration of a direct link between serial order in verbal WM and spatial attention: Retrieving a WM item induced (covert) attention shifts based on its WM position. This finding prompts the intriguing notion that serial order in verbal WM is intrinsically spatial in nature, such that maintaining serial order results in a spatially defined representation, and that spatial attention is involved in searching within this representation.

The current study opens new perspectives on the representation of serial order in WM by directly associating it with spatial attention. At the same time, it suggests new questions to be explored. First, whereas our results show a clear link with spatial attention at the level of retrieval (indicating a spatially defined representation), it remains to be determined whether spatial attention is involved also at the encoding level. Second, future research should explore to what extent the link between serial order and spatial attention proposed here is also involved in visuospatial WM and how this relation interacts with the more typical spatial-attention processes involved there. 
In conclusion, the current findings indicate that WM for serial order-even for nonspatially defined verbal items-is grounded in spatial attention. This result fits well with and broadens the scope of the general view that WM functions emerge from the directing of attention to long-term memory representations (Cowan, 1995; Postle, 2006), and it will challenge serial-order-coding theories to consider attention as a core ingredient. On a more general note, our results also indicate that the typically upheld separation between the verbal and spatial domains (e.g. Baddeley, 1986; Paivio, 1986) may sometimes prevent what may be a fruitful crossover of mechanisms from one domain to the other.

\section{Author Contributions}

J.-P. van Dijck and E. L. Abrahamse contributed equally in the development of the study concept, the collection and analyses of the data, and the drafting of the manuscript, and should be considered as joint first authors. All authors contributed to the study design and the interpretation of the results. S. Majerus and W. Fias provided critical revisions. All authors approved the final version of the manuscript for submission.

\section{Acknowledgments}

The authors thank Boris Ketels and Carsten Bundt for assisting with data collection.

\section{Declaration of Conflicting Interests}

The authors declared that they had no conflicts of interest with respect to their authorship or the publication of this article.

\section{Funding}

E. L. Abrahamse was supported by the Netherlands Organization for Scientific Research (Grant 446-10-025) and the Research Foundation Flanders (Grant 12C4712N). This work was supported by the Ghent University Multidisciplinary Research Partnership's Integrative Neuroscience of Behavioral Control Consortium and by Grant P7/11 from the Interuniversity Attraction Poles Program of the Belgian Federal Government.

\section{Note}

1. In Experiments 1 and 2, data from 1 participant were discarded because these participants' overall mean RTs and standard deviations were more than 2.5 standard deviations from the group means.

\section{References}

Awh, E., \& Jonides, J. (2001). Overlapping mechanisms of attention and spatial working memory. Trends in Cognitive Sciences, 5, 119-126.
Baddeley, A. (1986). Working memory. Oxford, England: Clarendon.

Baddeley, A. (2012). Working memory: Theories, models, and controversies. In S. T. Fiske, D. L. Schacter, \& S. E. Taylor (Eds.), Annual review of psychology (Vol. 63, pp. 1-29). Palo Alto, CA: Annual Reviews.

Burgess, N., \& Hitch, G. (1999). Memory for serial order: A network model of the phonological loop and its timing. Psychological Review, 106, 551-581.

Cowan, N. (1995). Attention and memory: An integrated framework (Vol. 26). New York, NY: Oxford University Press.

Fischer, M. H., Castel, A. D., Dodd, M. D., \& Pratt, J. (2003). Perceiving numbers causes spatial shifts of attention. Nature Neuroscience, 6, 555-556.

Henson, R. N. A. (1998). Short-term memory for serial order: The start-end model. Cognitive Psychology, 36, 73-137.

Johnston, J. C., McCann, R. S., \& Remington, R. W. (1995). Chronometric evidence for two types of attention. Psychological Science, 6, 365-369.

Majerus, S., Attout, L., D’Argembeau, A., Degueldre, C., Fias, W., Maquet, P., . . . Balteau, E. (2012). Attention supports verbal short-term memory via competition between dorsal and ventral attention networks. Cerebral Cortex, 22, 1086-1097.

Majerus, S., Heiligenstein, L., Gautherot, N., Poncelet, M., \& Van der Linden, M. (2009). Impact of auditory selective attention on verbal short-term memory and vocabulary development. Journal of Experimental Child Psychology, 103, 66-86.

Marshuetz, C. (2005). Order information in working memory: An integrative review of evidence from brain and behavior. Psychological Bulletin, 131, 323-339.

Nobre, A. C., Coull, J. T., Maquet, P., Frith, C. D., Vandenberghe, R., \& Mesulam, M. M. (2004). Orienting attention to locations in perceptual versus mental representations. Journal of Cognitive Neuroscience, 16, 363-373.

Oberauer, K., \& Hein, L. (2012). Attention to information in working memory. Current Directions in Psychological Science, 21, 164-169.

Paivio, A. (1986). Mental representations: A dual coding approach. New York, NY: Oxford University Press.

Posner, M. I. (1980). Orienting of attention. Quarterly Journal of Experimental Psychology, 32, 3-25.

Postle, B. R. (2006). Working memory as an emergent property of the mind and brain. Neuroscience, 139, 23-38.

Pratt, J., Spalek, F., \& Bradshaw, J. L. (1999). The time to detect targets at inhibited and noninhibited locations: Preliminary evidence for attentional momentum. Journal of Experimental Psychology: Human Perception and Performance, 23, 730-746.

Theeuwes, J., Kramer, A. F., \& Irwin, D. (2011). Attention on our mind: The role of spatial attention in visual working memory. Acta Psychologica, 137, 248-251.

van Dijck, J.-P., \& Fias, W. (2011). A working memory account for spatial numerical associations. Cognition, 119, 114-119. 